\title{
Mechanics considerations for microporous titanium as an orthopedic implant material
}

\author{
Sarah Thelen, ${ }^{*}$ François Barthelat, L. Catherine Brinson \\ Mechanical Engineering Department, Northwestern University, Technological Institute, 2145 Sheridan Road, Evanston, \\ Illinois 60208-3111
}

Received 30 July 2002; revised 30 September 2003; accepted 1 October 2003

Published online 28 April 2004 in Wiley InterScience (www.interscience.wiley.com). DOI: 10.1002/jbm.a.20100

\begin{abstract}
This article investigates mechanics issues related to potential use of a recently developed porous titanium (Ti) material for load-bearing implants. This material may have advantages over solid $\mathrm{Ti}$ of enhancing the bone-implant interface strength by promoting bone and soft tissue ingrowth and of reducing the bone-implant modulus mismatch, which can lead to stress shielding. Experimental data from ultrasound experiments and uniaxial compression testing on microporous $\mathrm{Ti}$ are presented. Analytic models to predict its elastic modulus and Poisson's ratio are discussed, including "structural" approaches (Gibson and Ashby's cellular solids) and a "composite material" approach (MoriTanaka). Finally, two-dimensional finite element models
\end{abstract}

based on optical micrographs of the material are presented. Simulations were performed for different conditions and levels of approximation. Results demonstrate that simple analytic models provide good estimates of the elastic properties of the porous $\mathrm{Ti}$ and that the moduli can be significantly reduced to decrease the mismatch between solid $\mathrm{Ti}$ and bone. The finite element simulations show that bone ingrowth will dramatically reduce stress concentrations around the pores. (C) 2004 Wiley Periodicals, Inc. J Biomed Mater Res 69A: 601-610, 2004

Key words: porous; titanium; micromechanics; finite elements; mechanical properties

\section{INTRODUCTION}

Metallic orthopedic implants have been used for decades for skeletal repair throughout the body. Issues including strength of the implant-bone interface and stress-shielding effects are important in the choice of the implant material and design; thus, much effort has been directed toward these areas. ${ }^{1}$

One of the key issues for a durable and successful implant is a strong bone-implant interface. It has been observed that smooth implant surfaces can cause encapsulation and lead to loosening of the implant. ${ }^{2}$ In an effort to promote long-term interface strength, porous coatings and materials have been developed. ${ }^{1,3} \mathrm{~A}$ porous coating or material can promote partial to complete bone ingrowth, which enhances the strength of the interface and reduces the capsule formation around the implant.

Another critical issue in many load-bearing im-

\footnotetext{
*Present address: Zimmer, Inc., 1800 West Center Street, Warsaw, IN 46581-0708

Correspondence to: L. C. Brinson; e-mail: cbrinson@ northwestern.edu

Contract grant sponsor: National Science Foundation
}

(C) 2004 Wiley Periodicals, Inc. plants is "stress shielding." 4,5 It is well known that bone regeneration and repair are promoted by mechanical loads. $^{4,6-9}$ A metal such as titanium (Ti), widely used for bone implants, is much stiffer than bone. An implant of solid Ti therefore can carry a disproportionate amount of the biological loads. The surrounding bone is then "stress shielded" and experiences abnormally low levels of stress, which can lead to resorption of the bone and again, loosening of the implant. ${ }^{10}$

In this article, novel porous commercially pure (cp)-Ti and Ti-6Al-4V materials are investigated as possible bone implant materials (see Fig. 1). Compressed bubbles of argon have been used to superplastically expand cp-Ti and Ti-6Al-4V up to porosities of 50\%. ${ }^{11,12}$ Furthermore, the pores can be elongated by applying a tensile load during the process ${ }^{12}$ and morphology controlled by suitable choice of powder precursor diameters. The advantages of this material for bone implant applications are multifold. A significant amount of the porosity is open, thus substantial bone ingrowth may be possible, which could greatly improve the bone-implant interface and may allow for efficient soft tissue attachment. Additionally, by increasing the porosity of the implant material its stiffness decreases correspondingly, reducing the stressshielding effect. 


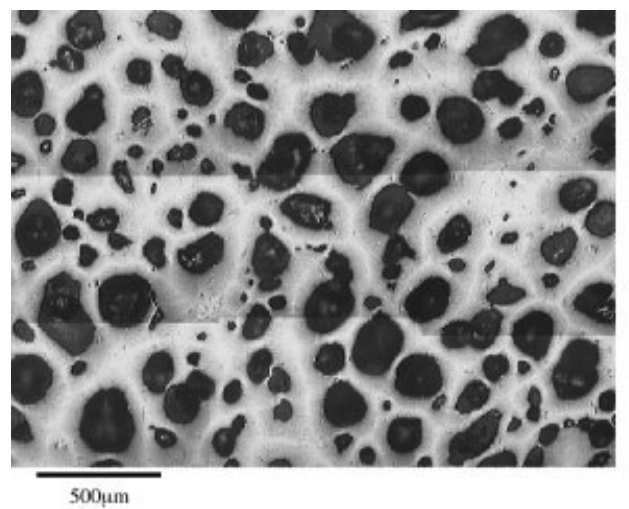

(a)

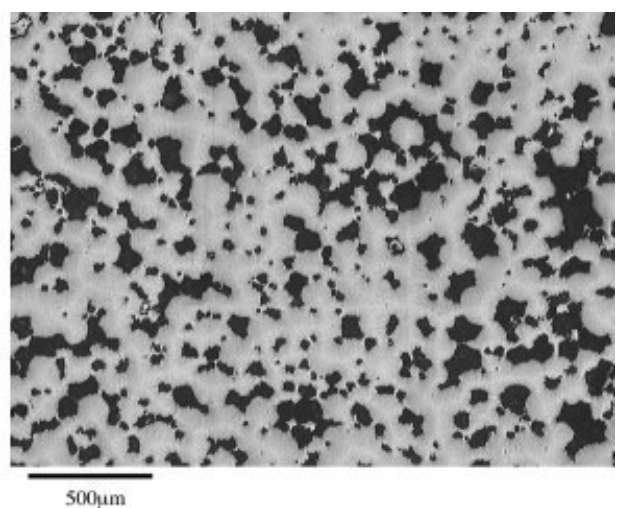

(b)

Figure 1. Micrographs of (a) $33 \%$ porous cp-Ti with predominantly spherical pores, and (b) $20 \%$ porous Ti-6Al-4V where cusp-like features are predominant.

To pursue the development of novel porous $\mathrm{cp}$-Ti or Ti-6Al-4V implants in structural applications, two major issues need to be addressed with respect to the material: the effect of the pore size and shape on 1 . the mechanical behavior of the material, and 2 . bone ingrowth.

Initial exploration into the first issue is the focus of this article, whereas the second issue is being studied with collaborators and results will be presented elsewhere. ${ }^{13-15}$ It has been shown that pore size and interconnectivity between the pores have a strong influence on bone ingrowth, ${ }^{16-18}$ although the effect of pore microstructure on bone and soft tissue ingrowth has not been significantly explored. Research into the effects of pore morphology and interconnectivity of this porous $\mathrm{Ti}$ on bone cell ingrowth is the focus of the companion studies.

The introduction of pores into a structural material will dramatically change its mechanical properties of stiffness, strength, and fatigue resistance. Thus, although the anticipated stiffness reduction can be beneficial to reduce stress shielding, the effect must be quantified and understood. In addition, the geometric microstructure of the porosity can be controlled by processing methods, therefore the effect of pore size, morphology, and spatial distribution on the local and global mechanical response needs to be assessed. Experimental methods, analytic and finite element modeling can be used to explore the effect of these parameters and optimize the morphology for orthopedic applications. This investigation therefore conducts initial studies on the mechanical properties of this new porous Ti material to begin documenting its properties for clinical use.

In this article, both experimental tests and modeling are pursued for porous cp-Ti and Ti-6Al-4V. Experiments include ultrasonic testing for elastic modulus as well as compressive loading well into the nonlinear deformation regime. Fatigue response, which may be adversely affected by porosity is not addressed in this report. The moderately porous $\mathrm{Ti}(35-50 \%$ porosity) lies at a relatively unexplored porosity level with respect to mechanical property modeling. Therefore, we examine several approaches. The abilities of foam mechanics models (intended for high porosity) ${ }^{19}$ and of a composite material model (intended for low porosity) ${ }^{20}$ to describe the elastic compressive behavior of these samples are explored and presented. Additionally, although analytic models for average properties are useful because of their speed and simplicity, they do not provide the flexibility and details of discretized finite element models. Therefore, several two-dimensional (2D), plane-strain finite element models based on micrographs of porous $\mathrm{Ti}$ samples were generated and analyzed. An initial finite element investigation into the effect of bone ingrowth on the local and global mechanical properties of the foam is also presented. The results present the mechanical properties of this new microporous Ti material, demonstrate the applicability of several types of models, and present important findings on possible effects of bone ingrowth on mechanical properties.

\section{EXPERIMENTAL PROCEDURE}

Both ultrasonic testing for elastic moduli and compressive mechanical loading to obtain complete stress-strain response were performed for several porous $\mathrm{Ti}$ specimens. Experimental procedure and results of each method are presented below.

\section{Ultrasound experiment}

The ultrasound method was used to determine the elastic properties. The ultrasound technique is ideal for highly po- 


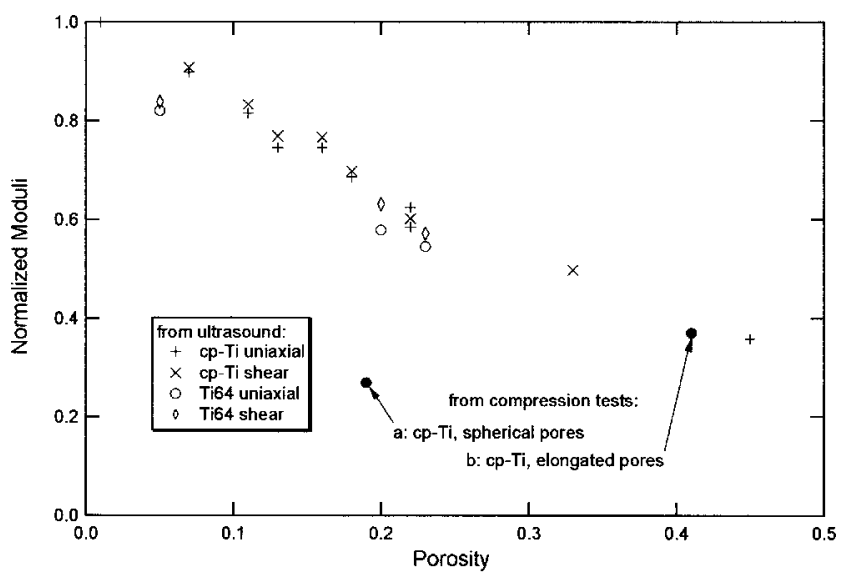

Figure 2. Normalized Young's moduli and shear moduli of porous $\mathrm{cp}-\mathrm{Ti}$ and $\mathrm{Ti}-6 \mathrm{Al}-4 \mathrm{~V}$ as a function of porosity obtained from ultrasound testing (normalized by $E=112 \mathrm{Gpa}$ and $E=117 \mathrm{Gpa}$ for cp-Ti and Ti-6Al-4V, respectively; shear modulus $42 \mathrm{Gpa}$ for both materials). Compression data from specimens $a$ and $b$ are discussed with Figure 3.

rous materials because deformations of the material caused by the ultrasound energy are sufficiently small such that plasticity can be avoided even in very narrow "struts" of the foam (see Fig. 1).

For this experiment, the specimen used was a porous $\mathrm{Ti}$ cube of about $10 \mathrm{~mm}$ in size. A transducer transmitted a sinusoidal signal to the front surface of the specimen and recorded the waves reflected by the opposite surface. The wave velocity was given directly by the delay between emitted and received signals and the sample dimensions. The wavelengths were chosen to be smaller than the dimension of the specimen, but greater than the size of the pores, to minimize scattering effects. ${ }^{21}$ Transducers of $2.5,5$, and 50 $\mathrm{MHz}$ were used.

The experiment used longitudinal waves (velocity $V_{\mathrm{L}}$ ) and shear waves (velocity $V_{\mathrm{S}}$ ) to determine the Young's and shear modulus of each specimen. The porous metal samples were confirmed to be isotropic and the Lamé constants for the material were found using ${ }^{22}$ :

$$
\mu=V_{S}^{2} \rho, \lambda=\left(V_{L}^{2}-2 V_{S}^{2}\right) \rho
$$

The density $\rho$ indicates the porous material density, as measured by the Archimedes method. The density was also used to calculate the porosity: $\Phi=\left[1-\left(\rho / \rho_{\mathrm{S}}\right)\right]$, where $\rho_{\mathrm{S}}$ is the density of the solid $\left(\rho_{\mathrm{S}}=4500 \mathrm{~kg} / \mathrm{m}^{3}\right.$ for $\mathrm{cp}$-Ti and $\rho_{\mathrm{S}}=4430$ $\mathrm{kg} / \mathrm{m}^{3}$ for Ti-6Al-4V).

\section{RESULTS}

Specimens of cp-Ti and Ti-6Al-4V with various porosities were tested. Variation of each modulus with orientation did not exceed $3.7 \%$ for any of the tested samples, confirming isotropy. The calculated moduli were normalized with respect to the modulus of the respective solid material (cp-Ti or Ti-6Al-4V), shown in Figure 2.
As expected, the moduli and porosity are inversely proportional. Despite what can be dramatic differences in microstructures (Fig. 1), the normalized moduli of the cp-Ti and Ti-6Al-4V samples follow a similar trend. The slightly lower moduli of the Ti-6Al-4V samples may be due to the shape of their pores: whereas the pores of the cp-Ti foams were roughly spherical and smooth, the pores of the Ti-6Al-4V foam showed many cusp-like features. Using the Young's and shear moduli, the Poisson's ratios were calculated and are shown later in Figure 8(b). The Poisson's ratios were not found to vary significantly with the porosity.

\section{Compressive experiments}

Uniaxial compressive tests were performed on porous $\mathrm{Ti}$ specimens by Davis et al. ${ }^{12}$ The specimens were subjected to a compressive load in a screwdriven load frame and the displacements of the platens were monitored using a laser extensometer. From the load and the initial sample dimensions, the compressive stresses were computed and from the displacement and the initial gauge length, the strains were computed.

The resulting stress-strain curves are shown in Figure 3 for a $19 \%$ porous $\mathrm{cp}$-Ti containing round pores (specimen $a$ ) and a $41 \%$ porous $\mathrm{cp}-\mathrm{Ti}$ specimen containing pores elongated along the loading direction (specimen $b$ ).

These stress-strain curves are typical of porous metals in compression: they exhibit an initial deformation, a plateau region corresponding to collapse of the pores, and an inflection upward corresponding to densification. The initial moduli (at strain levels $<0.5 \%$ ) taken from these curves for specimens $a$ and $b$ are 30 and $41 \mathrm{Gpa}$, respectively (normalized moduli are 0.27 and 0.37 ). The difference in the initial moduli can be attributed to the pore structure because elongation in the loading direction (specimen b) should stiffen the material response. (See also finite element results in the section Finite Element Method.)

The normalized moduli from the compression tests are plotted along with the results of the ultrasonic tests in Figure 2, illustrating the discrepancies. The normalized initial modulus for specimen $a$ from compression test data is significantly lower than that indicated by the ultrasound method for samples of similar porosities. The normalized initial modulus measured for specimen $b$ (.37 from compression data in Fig. 3), appears to agree with the results shown in Figure 2. However, because the pores of specimen $b$ are elongated in the loading direction, whereas the pores of the samples of Figure 2 are approximately spherical, specimen $b$ should exhibit a higher modulus for the same porosity compared with Figure 2 . Thus, both 


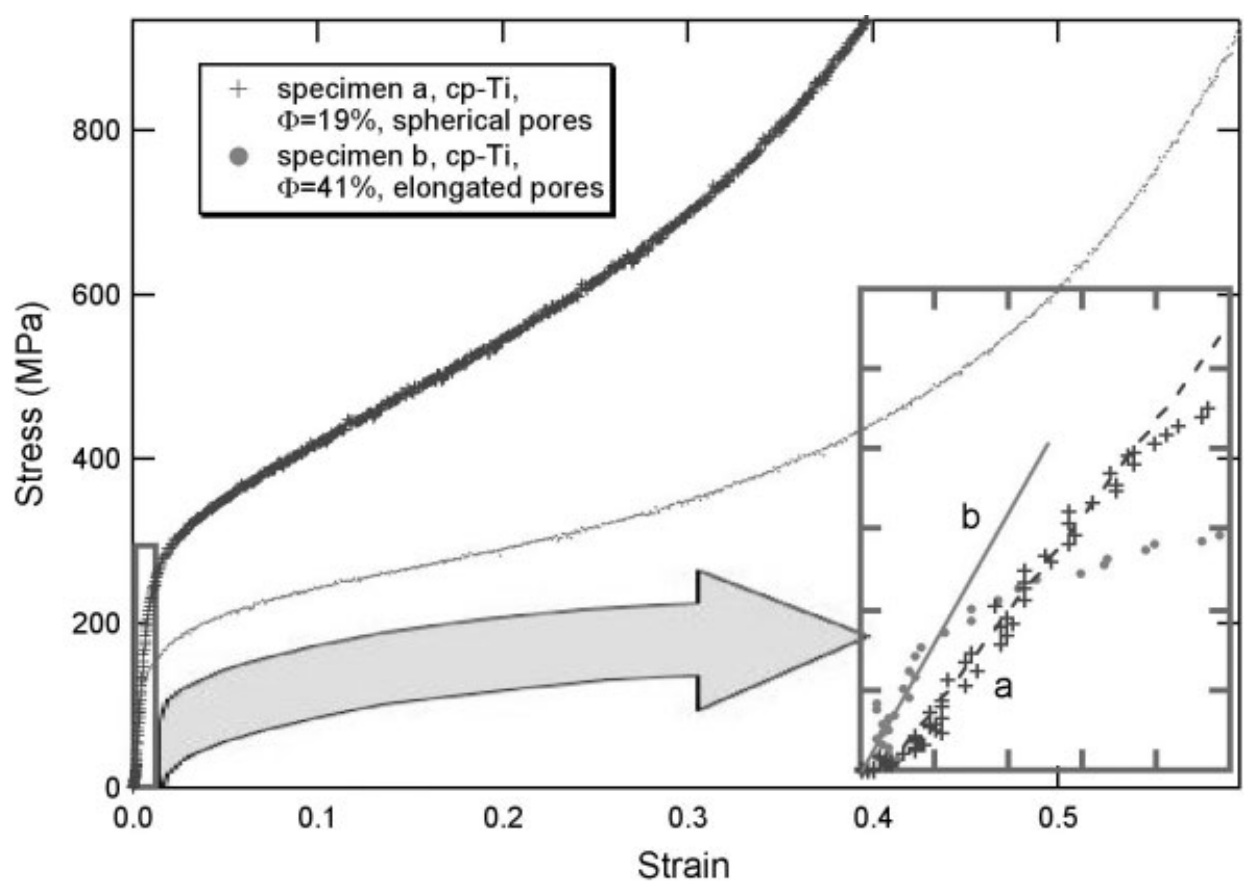

Figure 3. Compression stress-strain curve of two porous cp-Ti samples: specimen $a$ (19\% porous) and specimen $b$ (41\% porous, elongated pores). Inset shows exploded view of data to 0.01 strain level and fits for moduli.

compressive tests indicate lower initial moduli than the ultrasound testing. These lower values can be explained by localized damage accumulating at very low strain levels in compression testing, in the region where the stress-strain curves appear linear, making the material effectively softer. This concept is discussed further in Comparison of the Methods and Discussion. These results confirm the preference for the ultrasonic method to determine elastic properties as its infinitesimal deformations preclude plasticity influences.

\section{ANALYTIC METHODS}

In this section, we discuss the analytical methods used to investigate the mechanical properties of the porous Ti material. The methods fall into two broad categories: models based on a cellular network structure ("structural models" developed for high porosity) and micromechanics models developed originally for composite materials ("composite models" appropriate for low porosity), most notably the Mori-Tanaka method. Here, each of the models is discussed and the calculated moduli are presented as a function of material porosity. The architecture of the material and its effects on mechanical properties are also discussed. In Comparison of the Methods and Discussion section, the results of these analytic methods are compared with the results of the finite element analysis and experimental studies. Appropriate use of each of the modeling strategies for the porous Ti is discussed.

\section{Gibson et al. "structural" models}

These models were developed for foams and cellular materials ${ }^{19}$ and are based on the analysis of a unit cell in the material, derived from approximate geometry. The unit cells are analyzed using simple beam theory and the global response of a repeating array of identical unit cells inferred. The first assumption made in these models is that the walls within the porous samples are thin, or equivalently that the porosity is above approximately $70 \%$. The second assumption is that a single unit cell is representative of the whole structure, which is therefore assumed to be without defects.

The simplest structural model is the 2D honeycomb, where the unit cell is hexagonal. Although this model is $2 \mathrm{D}$, it can be used to approximate more complicated cellular solids. The applicable expression derived from beam theory and experiments is:

$$
E^{*} / E=2.3(t / l)^{3}
$$

where $E^{*}$ is the Young's modulus of the porous material, $E$ is the Young's modulus of the solid material, $t$ and $l$ are the thickness and the length of the walls, respectively. Using geometric arguments, Equation (2) 


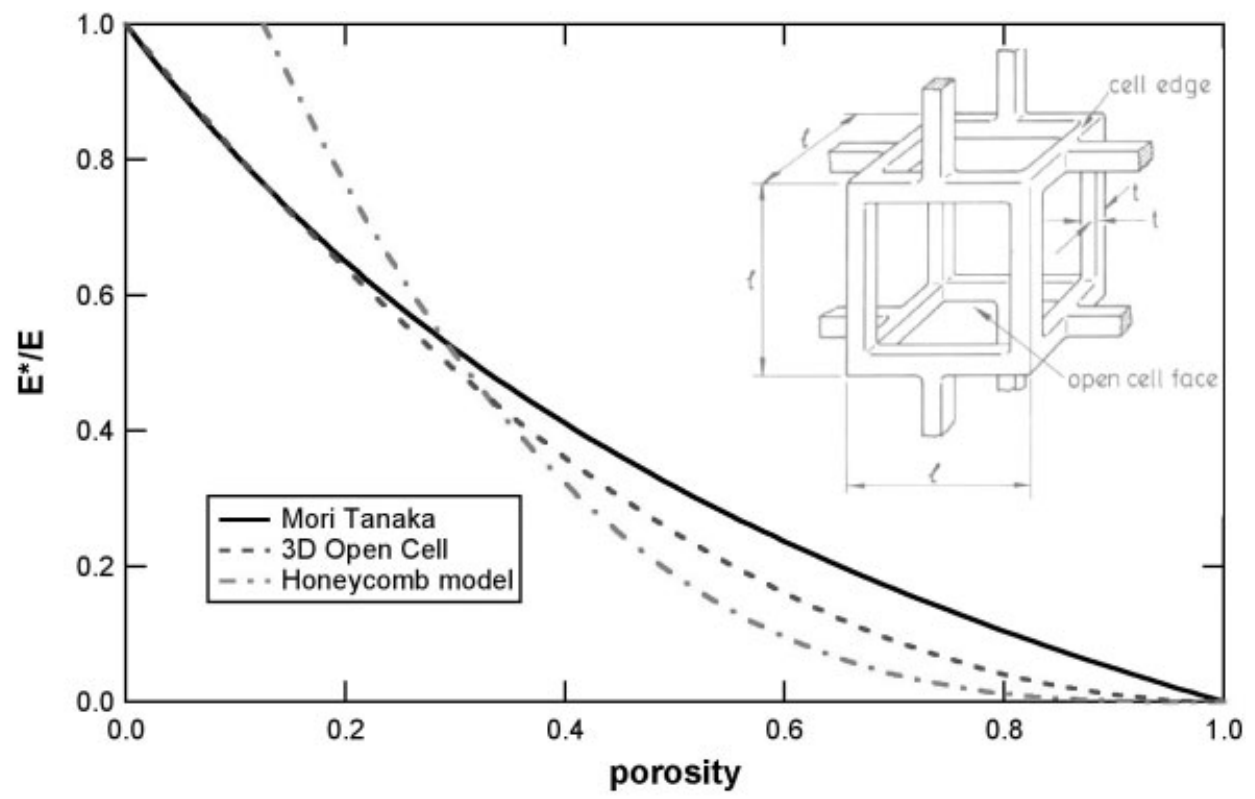

Figure 4. Predictions for moduli using the three analytic models presented; inset is 3D open cell model from Gibson and Ashby ${ }^{19}$ where loading direction is vertical.

can be rewritten in terms of porosity, where $\Phi$ is the porosity. ${ }^{19}$

$$
\frac{E^{*}}{E}=2.3\left(\frac{\sqrt{3}}{2}(1-\Phi)\right)^{3}
$$

To model a 3D open cell material, Gibson and Ashby ${ }^{19}$ used the unit cell shown as an inset in Figure 4 to obtain:

$$
\frac{E^{*}}{E}=(1-\Phi)^{2}
$$

The normalized moduli calculated by these two approaches are plotted in Figure 4 as a function of porosity.

The simple honeycomb model, which assumes porosity $>70 \%$, gives unrealistic predictions for behavior of materials with porosity $<20 \%$. Thus, further investigation of this model was not pursued. The 3D open cell model is also based on assumptions of high porosity; however, experiments have shown that this model provides good predictions of modulus for materials with porosities ranging from 10 to $90 \%{ }^{19,23}$ Hence, we compared results of the 3D open cell approach to experimental, other analytic, and finite element analysis results for all porosity levels of the $\mathrm{Ti}$ material in the Conclusion secion.

\section{Mori-Tanaka "composite material" approach}

The Mori-Tanaka method was originally developed for two-phase composites, with random inclusions of any shape $\mathrm{e}^{20,24-26}$ and has been the basis of many micromechanics calculations. In the case of a porous material, the pores are treated as null-stiffness inclusions and although developed for lower volume fractions this method has been successfully utilized for materials with up to $50 \%$ porosity. ${ }^{24,25}$ The method is derived assuming that the average strain in the pores can be approximated by that of a single pore in the matrix subjected to the (unknown) average matrix strain. A recursive relationship is used along with the analytical solution for a single pore, based on the Eshelby tensor, ${ }^{27,28} \mathbf{S}$, which is dependent on pore shape.

Using this approach and neglecting any internal pressure within the pores, the expression for the effective modulus of a porous material can be reduced to

$$
C^{*}=C\left[1-\frac{\Phi}{(1-\Phi) S^{-1}+\Phi}\right]
$$

where $C^{*}$ is the composite bulk or shear modulus, $S^{-1}$ is the inverse of the Eshelby tensor, and $\Phi$ is the porosity of the material. The normalized stiffness predicted by the Mori-Tanaka approach for the case of spherical voids is compared with the results predicted by the analytic structural models in Figure 4.

\section{FINITE ELEMENT METHOD}

Two- and three-dimensional finite element models have been previously used to study other porous and cellular materials. ${ }^{19,29-32}$ These simulations can include 


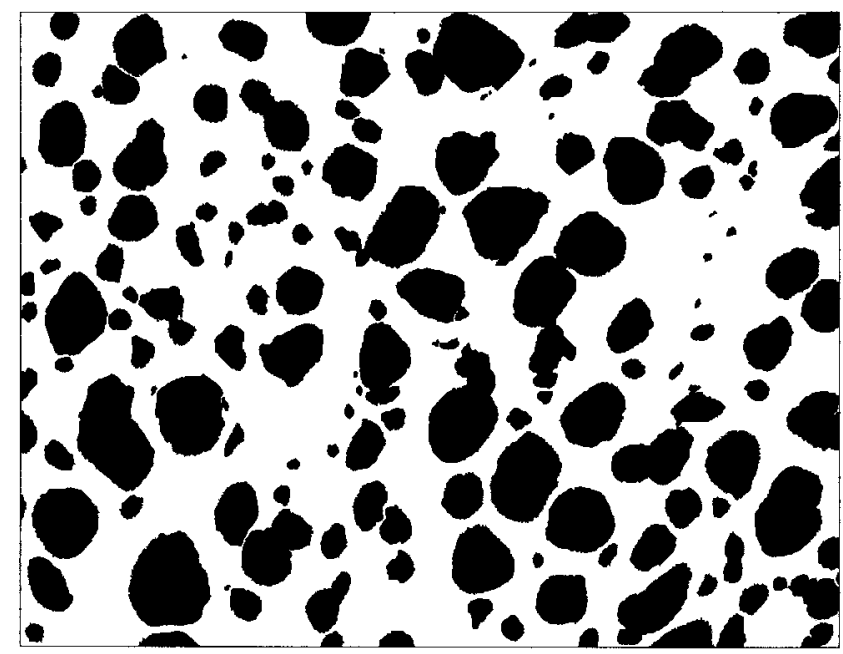

(a)

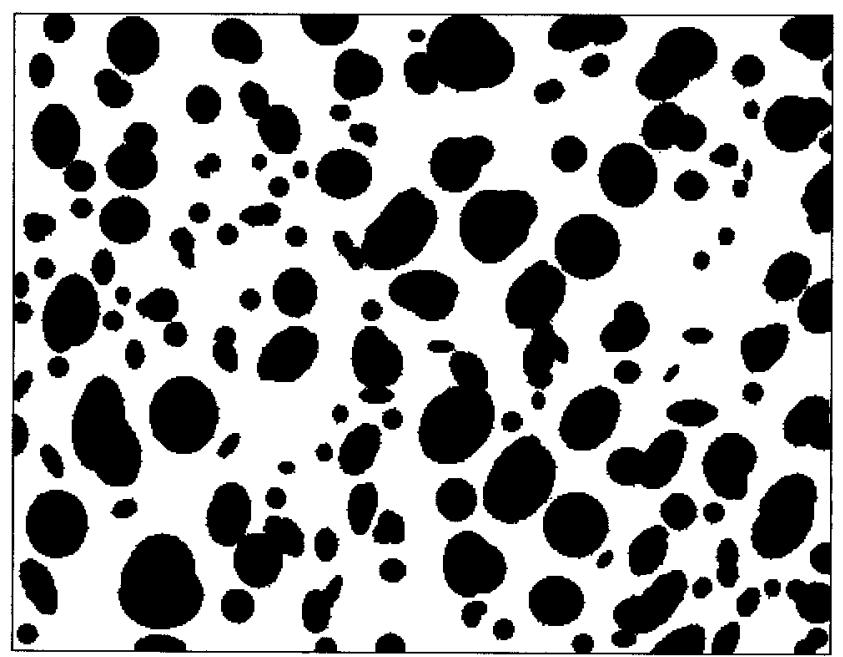

(b)

Figure 5. Binary image of the $33 \%$ porous $\mathrm{cp}-\mathrm{Ti}$ and its simplified geometry, where the pores have been approximated by ellipses ( $x$ direction is to the right).

parameters that the previously discussed analytical models do not take into account. For example, numerical results showed that the distribution of the pores (random or regular distribution), and the presence of defects (missing walls, non-uniform thickness or waviness of the walls, randomly filled cells) had a significant impact on the overall mechanical response of the material. ${ }^{19,29-34}$ Here, we perform finite element simulations from discretized images of porous $\mathrm{Ti}$ microstructure and consider the effects of pore geometry and bone ingrowth on mechanical properties and local stresses. Comparison to analytic approaches appears in Conclusion.

\section{Model generation}

In this work, 2D finite element models of the material at different porosity levels were generated and solved using elastic elements. A 2D model of a more complex 3D solid has limitations on its accuracy. However, literature has shown that many aspects of material response can be reasonably represented, in particular for quasistatic elastic response. ${ }^{35,36}$ In addition, 2D models can be effectively used to gain understanding of the influence of different modeling parameters on results and to rank microstructures relative to one another. Finally, in comparison to most finite element simulations that are based on artificially generated microstructures, here we are able to retain and mesh the precise geometry obtained from micrographs of porous Ti samples. Thus, the results in this section represent a powerful step beyond the analytical models of the previous sections because they represent the microstructure accurately and can present valuable information on local stress and strain states.

To build the finite element models, micrographs of the surface of the specimens used for the ultrasonic experiment were first obtained and converted into binary images (compare Figs. 1 and 5). The area of the micrograph in these figures is approximately $15 \%$ of the entire specimen surface area. This surface is representative of the entire area, validated by simulations with different areas. Similar micrographs of specimens with various porosities for both $\mathrm{cp}-\mathrm{Ti}$ and $\mathrm{Ti}-$ $6 \mathrm{Al}-4 \mathrm{~V}$ were constructed. The software OOF (ObjectOriented Finite Element Analysis ${ }^{37}$ ) was used to mesh the surface in each case with plane-strain triangular elements, differentiating the solid matrix from the pores. The solid matrix elements were assigned the isotropic properties of $\mathrm{cp}-\mathrm{Ti}$ or $\mathrm{Ti}-6 \mathrm{Al}-4 \mathrm{~V}$. The elements within the voids were assigned a modulus close to zero (five orders of magnitude lower than the surrounding matrix).

\section{Unmodified simulations}

A small unidirectional strain $(0.05 \%)$ was applied in the vertical direction (along the $y$ axis). The resulting overall stress was obtained by dividing the sum of the loads on the top nodes by the width of the top surface (depth is unity). The meshes were refined until convergence was achieved. From the plane-strain Young's modulus $E^{\prime}$ and Poisson's ratio $v^{\prime}$, the Young's modulus $E$ and Poisson's ratio $v$ were calculated using:

$$
E=E^{\prime}(1-v) \text { and } v=v^{\prime} /\left(1+v^{\prime}\right)
$$

Similar simulations were performed by loading the model horizontally (along the $x$ axis).

The resulting moduli and Poisson's ratios for differ- 


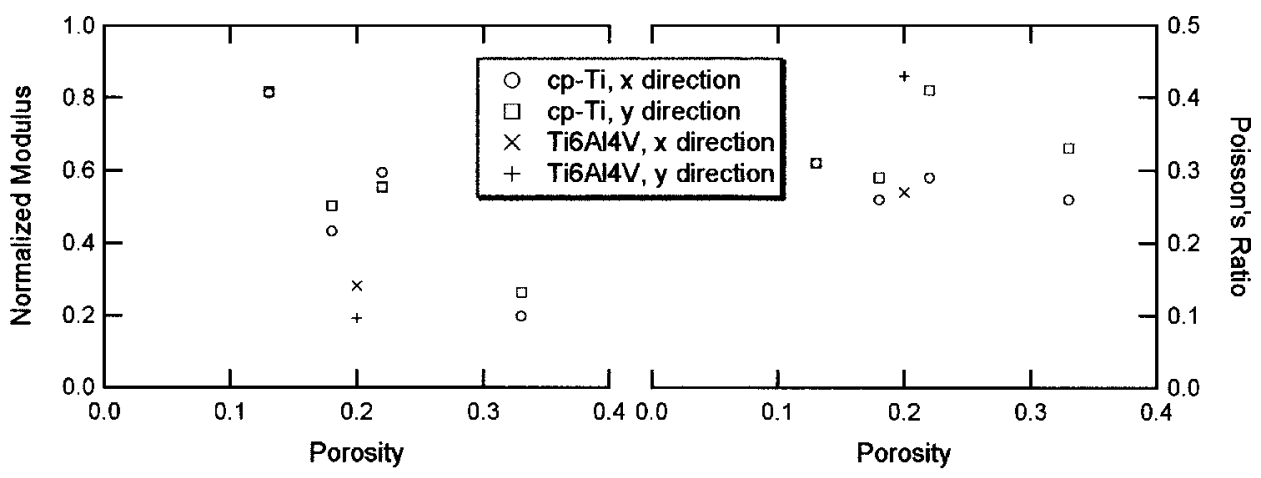

Figure 6. Normalized moduli and Poisson's ratios from the finite element simulations.

ent porosities and directions are shown in Figure 6. The results demonstrate anisotropic behavior, with anisotropy ratios up to 1.3 for the $33 \%$ porosity $\mathrm{cp}-\mathrm{Ti}$ model.

\section{Geometric and bone-filling modifications}

From the $33 \%$ porosity cp-Ti model, the importance of morphology changes and bone ingrowth on the mechanical properties were examined. The cases considered are: 1 . pore smoothing, 2 . elongated pores, and 3 . bone ingrowth. In the first case, the shapes of the pores were approximated with ellipses, removing some of the detailed features of the pore shapes (Fig. $5)$. For the second case, pores were elongated by stretching the height by a factor of 1.5 . For bone ingrowth case, elements in the pores were assigned material properties simulating bone $(E=17 \mathrm{GPa}, v=$ $\left.0.41^{18}\right)$. In one simulation, all pores were completely filled with bone material. In a second simulation only an outer area, $35 \%$ of the total pore area of the model, was filled, representing partial bone ingrowth.

Figure 7 shows the results of the finite element simulations for these modifications. Only moderate

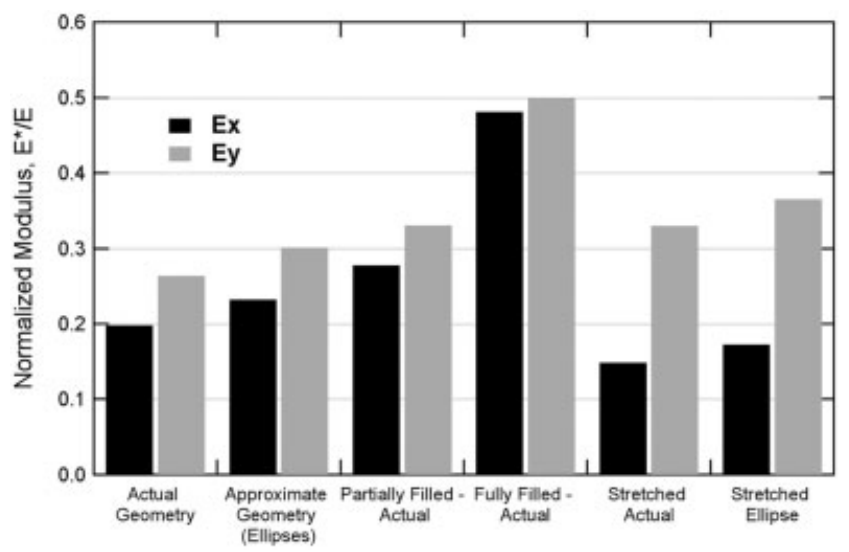

Figure 7. Elastic moduli found by finite element simulations using different $33 \%$ cp-Ti models. changes in modulus from the "actual geometry" case are seen for smoothing pore surfaces ("ellipses" case) or for the $35 \%$ bone-filled simulation. Elongating the pores has the result of increasing the modulus in the

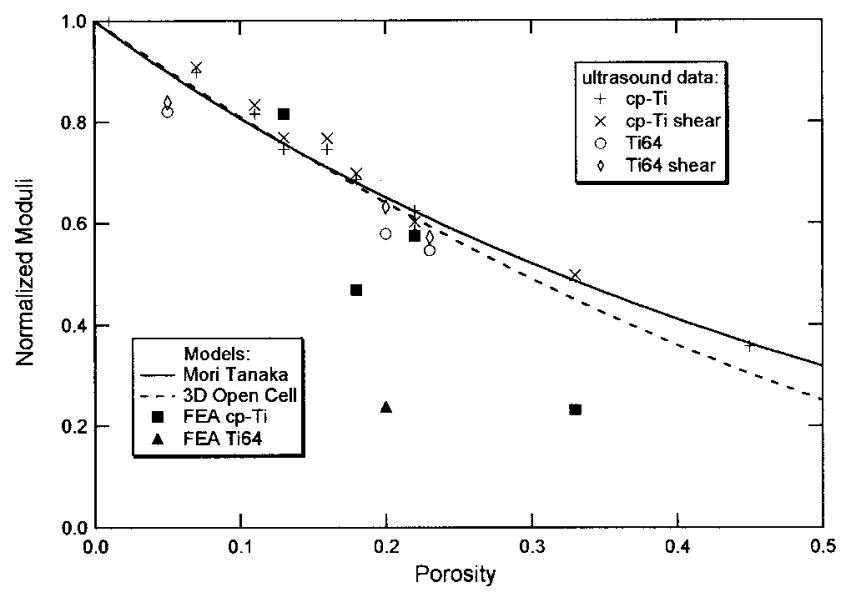

(a)

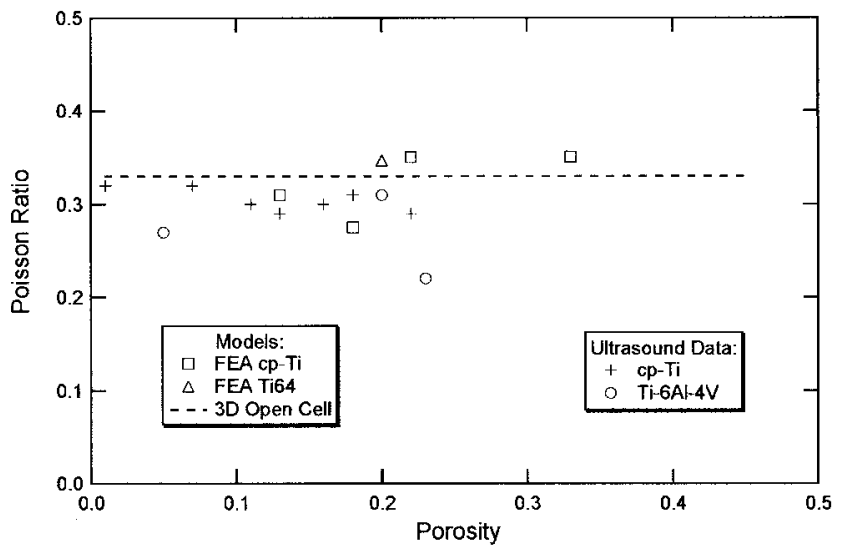

(b)

Figure 8. (a) Normalized moduli experimentally measured by ultrasound, and as predicted by Mori-Tanaka, Open Cell model, and finite element analyses (FEA are average of $x$ and $y$ directions). (b) Poisson's ratios experimentally measured by ultrasound and as predicted by the Open Cell model and finite element analyses (FEA results averaged for $x$ and $y$ directions). 


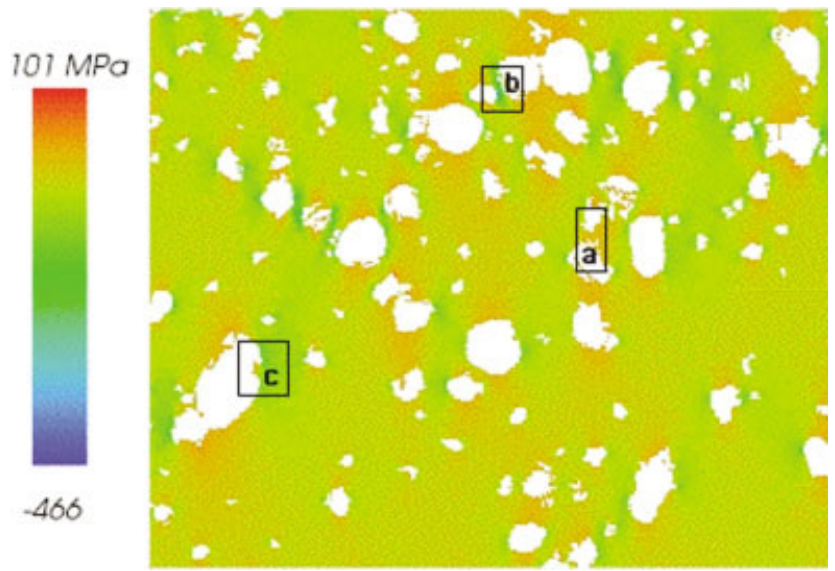

Figure 9. Sample section of stress contours predicted by finite elements for sample with irregularly shaped, rough pores. Note areas where pores could be consolidated [e.g., (a) and (b)], and note that the rough projections of pores to the interior carry no load (c). Load vertical.

elongation direction with a corresponding reduction in the transverse direction. Thus, fabrication techniques that allow pore elongation ${ }^{11}$ can be fruitfully pursued to tailor implant stiffness in specific directions. The properties for materials with complete bone ingrowth are striking, with a modulus double that of the baseline material.

\section{COMPARISON OF THE METHODS AND DISCUSSION}

In Figure 8(a,b), the moduli and Poisson's ratios found by ultrasonic experiments and the analytic and finite element approaches are presented. Both the 3D open cells model and the Mori-Tanaka approach agree well with the ultrasound experimental results for the Young's modulus.

The finite element simulations did not consistently reproduce the experimental data for the Young's mod-

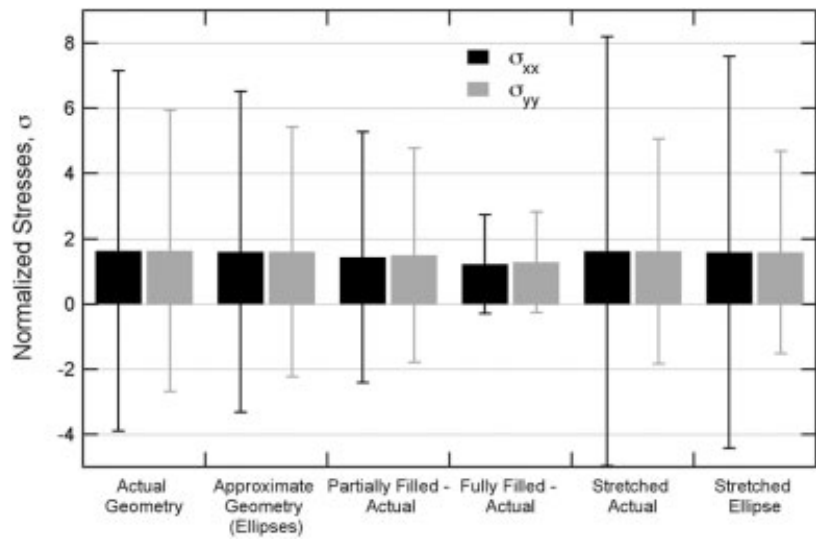

Figure 10. Comparison of normalized stresses for variations of the $33 \%$ porous cp-Ti sample images. Mean stresses are the solid bars, peak stresses are the error bars. From left to right, the stresses used to normalize the plotted values for the $x$ direction are as follows: $-11.9,-11.3,-13.9,-16.9$, $-30.6,-8.6$, and $-10.0 \mathrm{MPa}$. From left to right, the stresses used to normalize the plotted values for the $y$ direction are as follows: $-16.6,-15.3,-18.8,-20.6,-32.1,-21.6$, and $-23.6 \mathrm{MPa}$

ulus. The finite element predictions for the 13 and $22 \%$ porous samples agree well with the ultrasound and analytic results; however, the 2D finite element analysis significantly underestimated the moduli for the remainder. The finite element simulations also predicted moderately anisotropic behaviors, where the experimental results showed that the specimens were isotropic. One reason for the observed discrepancies may be the simplification of the complex 3D structure to a $2 \mathrm{D}$ plane-strain model. Because the microstructure is a complex 3D structure, it is not necessarily well represented by a single 2D image.

Additionally, a closer look at the micrographs of the porous Ti specimens (Figs. 1 and 9) suggests that the complex microarchitecture of the material may not be captured fully by the percent porosity alone. Certain features decrease the porosity value of the material, yet in $2 \mathrm{D}$ they transmit minimal load and/or are

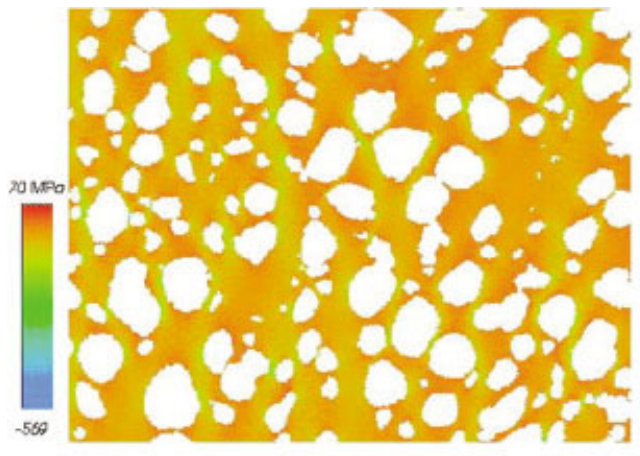

(a)

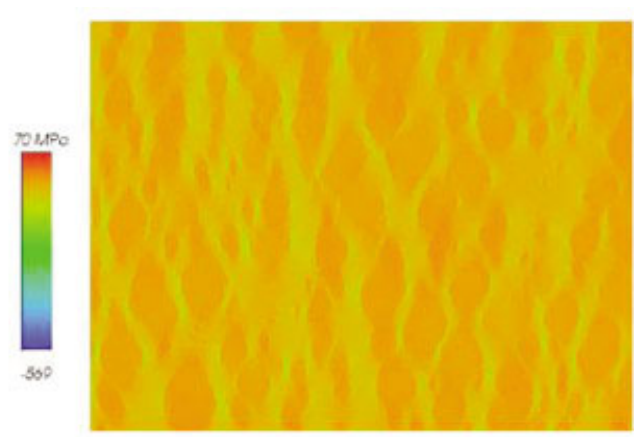

(b)

Figure 11. Stress contours for $\sigma_{\mathrm{yy}}$ when loaded in the $y$ direction, for the case of the actual microstructure (A) and microstructure filled with bone material (B). 
quickly stressed beyond yield. These features are termed "structurally insignificant" material. Considering Figure 9, roughness of the pores such as shown in box $c$, provides material that decreases the porosity of the specimen with minimal change in overall stiffness. In addition, when pores are very closely spaced, the small ligaments of material separating them either bear little load (box $a$ ) or are quickly stressed beyond the plastic limit (box $b$ ). In either case, tightly spaced pores may be considered to act essentially as a single larger pore. Thus, in cases containing rough and irregularly spaced pores, using the experimentally measured porosity for comparison to $2 \mathrm{D}$ finite element models may contribute to apparently underestimated predictions of the moduli. A more rigorous $3 \mathrm{D}$ technique to identify structurally inefficient material could be developed to provide an "effective porosity" measure more indicative of material response.

Despite the potential loss of accuracy in the absolute magnitude of the modulus, a 2D finite element analysis of the actual microstructure remains an effective method to obtain information on the global and localized stress and strain fields resulting from different microgeometries. In this article, the effects of smoothed pores, bone-filled pores, and elongated (stretched) pores were examined. Figure 10 shows the variations in mean nodal stress and peak nodal stress (defined as three standard deviations from the mean) for the different finite element models. The elongated pore case shows a significant increase in the stress localization. The results for the bone-filled pores are even more striking, where the values of the peak stresses decrease by more than a factor of 2, agreeing with previous data by Kohn et al. ${ }^{38}$ The dramatic change in moduli and stress concentrations for fully filled materials can be partially attributed to load sharing between the bone material and the $\mathrm{Ti}$ (as in composite materials) and to the increased support of the highly stressed portions of the metal. These results indicate that processing to ensure open porosity and sufficient pore interconnectivity is critical for porous Ti in bone implant applications, as implant durability will be greatly enhanced by bone ingrowth via local stress reductions. Notice that the average stresses in the sample remain largely unchanged for all cases. This can also be seen in Figure 11, showing stress color contours for the unfilled and filled cases.

\section{CONCLUSION}

Ultrasound experiments and analytic and numerical simulations were performed on porous Ti samples of different porosity and provided values for their Young's moduli and Poisson's ratios. Findings consistently indicate that a porous Ti material will decrease the material modulus, perhaps providing relief from the stress shielding phenomenon in bone implant applications. Among the analytic predictive approaches examined, both the open cell model and Mori-Tanaka analysis provided results consistent with the experimental values. The Mori-Tanaka approach has the advantage of being able to account for elongated pores and random orientations. Although based on micrographs of the actual structure of the specimens, the finite element models did not consistently reproduce the experimental values for modulus. This result is believed to be largely attributed to the $2 \mathrm{D}$ plane-strain approximation of a complex 3D microstructure. This finding suggests the need for further studies using tomographically produced 3D microstructures to fully capture the behavior of these materials.

However, the 2D finite element simulations have proven quite valuable to study the relative changes in properties resulting from changes in pore geometry or filling the pores with bone. The finite element results also provide approximate measures of the localized stress and strain field variations between different pore microstructures. These important results cannot be obtained by the analytic approaches. Here it was shown that once bone ingrowth is achieved, the localized stress concentrations are dramatically decreased, indicating potentially longer lifetime and decreased failure rate of an implant. The approximate results from the $2 \mathrm{D}$ analysis are also useful to screen microstructure options. Here it was shown that elongated pores can be effectively used to tailor stiffness in specific directions. In addition, because bone cell growth responds to mechanical loading, information on the local stress and strain at the surface of the pores, available from the FE studies, may prove useful to the biologists interested in the rate of bone cell growth in samples under moderate load.

However, further study of fully porous $\mathrm{Ti}$ is needed because results also indicate the presence of highly localized yielding of material well within the nominal elastic range, causing concern regarding implant durability and lifetime. Future work should assess local material response in more detail with finite element models incorporating plasticity and 3D microstructures coupled with careful experiments. Ultimately, such studies can be used to tailor the morphology of the pores for optimum modulus, strength, fatigue resistance, and cell adhesion.

The authors thank collaborators David Dunand and Sam Stupp for inspiration and discussion.

\section{References}

1. Engh CA, Bobyn JD. Biological fixation in total hip arthroplasty. Thorofare, NJ: SLACK; 1985. 
2. Chang, Y-S, Oka M, Kobayashi M, Gu H-O, Li Z-L, Nakamura $\mathrm{T}$, Ikada Y. Significance of interstitial bone ingrowth under load-bearing conditions: A comparison between solid and porous implant materials. Biomaterials 1996:17;1141-1148.

3. Greco RS, editor. Implantation biology: the host response and biomedical devices. Boca Raton, FL: CRC Press; 1994. p 418.

4. Jacobs JJ, Sumner DR, Galante JO. Mechanisms of bone loss associated with total hip replacement [Review]. Orthop Clin North Am 1993;24(4):583-590.

5. Amstutz HC, editor. Hip arthroplasty. New York: Churchill Livingstone; 1991.

6. Van Lenthe G, Waal Malefijt M, Huiskes R. Stress shielding after total knee replacement may cause bone resorption in the distal femur. J Bone Joint Surg Br 1997:79(1):117-122.

7. Rubash HE, Sinha RK, Shanbhag AS, Kim SY. Pathogenesis of bone loss after total hip arthroplasty [Review]. Orthop Clin North Am 1998;29(2):173-186.

8. Wan ZN, Dorr LD, Woodstone T, Ranawat A, Song M. Effect of stem stiffness and bone stiffness on bone remodeling in cemented total hip replacement. J Arthroplasty 1999;14(2):149159.

9. Santare M, Joshi M, Advani S. Analysis of a femoral hip prosthesis designed to reduce stress shielding. J Biomech. Submitted for publication.

10. Black J. Biological performance of materials, fundamentals of biocompatibility (3rd ed.). New York: Marcel Dekker; 1999.

11. Dunand DC, Teisen J. Superplastic foaming of titanium and Ti-6Al-4V. Mater Res Soc Symp Proc Southern Biomed Eng Conf Proc Biomater 1998;521:231-236.

12. Davis NG, Teisen J, Schuh C, Dunand DC. Solid-state foaming of titanium by superplastic expansion of argon-filled pores. J Mater Res 2001;16:1508-1519.

13. Spoerke ED, Stupp SI. Colonization of organoapatite-titanium mesh by pre-osteoblastic cells. J Biomed Mater Res 2003;67A: 960-969.

14. Spoerke ED, Murray NG, Li H, Brinson CL, Dunand DC, Stupp SI. Organoapatite-titanium foam: A bioactive composite for orthopedic tissue engineering. Nat Mater. 2003. Submitted for publiction.

15. Li H, Oppenheimer SM, Stupp SI, Dunand DC, Brinson LC. Effects of pore morphology and bone ingrowth on mechanical properties of microporous titanium as an orthopaedic implant material. Jpn Mater Trans 2004; in press.

16. Klawitter J, Hulbert S. Application of porous ceramics for the attachment of load bearing internal orthopedic applications. J Biomed Mater Res 1971;6(Symp 2, part 1):161-229.

17. Bobyn JD, Pilliar RM, Cameron HU, Weatherly GC. The optimum pore size for the fixation of porous-surfaced metal implants by the ingrowth of bone. Clin Orthop 1980;150:263-270.

18. Simske S, Ayers R, Bateman T. Porous materials for bone engineering. Mater Sci Forum 1997;250:151-182.

19. Gibson L, Ashby M. Cellular solids: Structure and properties. New York: Cambridge University Press; 1997.

20. Mori T, Tanaka K. Average stress in matrix and average elastic energy of materials with misfitting inclusions. Acta Metall 1973;21:571-574.
21. Wanner A. Elastic modulus measurements of extremely porous ceramic materials by ultrasonic phase spectroscopy. Stuttgart, Germany: Institut fur Metallkunde der Universitat Stuttgart; 1998.

22. Thelen S. Titanium foam for use in bone implants: Microstructure effect on effective properties and local stress states [MS Thesis]. Evanston, IL: Northwestern University; 2000.

23. Goods SH, Neuschwanger CL, Whinnery LL. Mechanical properties of a structural polyurethane foam and the effect of particulate loading. Mater Res Soc symp Proc 1998;521:15-20.

24. Weng D. Some elastic properties of reinforced solids, with special reference to isotropic ones containing spherical inclusions. Int J Eng Sci 1984;22(7):845-856.

25. Benveniste Y. A new approach to the application of MoriTanaka's theory in composite materials. Mech Mater 1987;6: 147-157.

26. Aboudi J. Mechanics of composite materials: a unified micromechanical approach. Amsterdam: Elsevier; 1991

27. Eshelby JD. The determination of the elastic field of an ellipsiodal inclusion, and related problems. Proc Royal Soc London 1957;A(241):376-396.

28. Mura T. Micromechanics of defects in solids. Boston, MA: Kluwer Academic; 1987.

29. Gans HD, Woodmansee PR. Improved method of modeling porous materials using finite elements. Comput Struct Mech Mater Biomater 1992;44:1055-1063.

30. Moorthy S, Ghosh S. Model for analysis of arbitrary composite and porous microstructures with voronoi cell finite elements. Int J Numer Methods Eng Metal Powder Rep 1996;39:23632398.

31. Hollister SJ, Brennan JM, Kikuchi N. A homogenization sampling procedure for calculating trabecular bone effective stiffness and tissue-level stress. J Biomech 1994;27(4):433-444.

32. Guo XE, McMahon TA, Keaveny TM, Hayes WC, Gibson LJ. Finite element modelling of damage accumulation in trabecular bone under cyclic loading. J Biomech 1994;27:45-55.

33. Gibson L, Ashby M. The mechanics of three-dimensional cellular materials. Proc Royal Soc London 1982;A(382):43-59.

34. Gibson L, Ashby M, Schajer G, Robertson C. The mechanics of two-dimensional cellular materials. Proc Royal Soc London 1982;A(382):25-42.

35. Shen H, Lissenden CJ. 3D finite element analysis of particlereinforced aluminum. Mater Sci Eng 2002;A338:271-281.

36. Skallerud B, Zhang ZL. Finite element modelling of cracked inelastic shells with large deflections: two-dimensional and three-dimensional approaches. Fatigue Fract Eng Mater Struct 2000;23(3):253-261.

37. National Institute of Standards and Technology. Object oriented finite element analysis. Gaithersberg, MD: National Institute of Standards and Technology.

38. Kohn DH, Ko CC, Hollister SJ. Stress analysis of porous coated total joint replacements: Coupled global/local modeling. Am Soc Mech Eng Bioengin Div (Publication) BED J Biomech 1992; 22:297-300 